It is proposed to discuss elsewhere the ways in which the Cluster Theory permits simple treatments of other physical properties of gases, such as specific heat, viscosity, light scattering, the Joule-Thomson effect, and the pressure-broadening of spectral lines.

$$
\text { C. F. GoOdeve. }
$$

Sir William Ramsay Laboratories of Inorganic and Physical Chemistry, University College,

$$
\text { London. }
$$$$
\text { June } 15 .
$$

${ }^{1}$ See, for example, O.Maass "Physical Chemistry" by 'l'aylor, vol. 1, p. 98 (1924); W. C. McC. Lewis "Kinetic Theory", p. 72 (1923); and Trashburn "Principles of Physical Chemistry", p. 38 (1921).

2 Fowler, "Statistical Mechanics" p. 291 (1936).

${ }^{3}$ Keesom, W. H., Phys. Z., 22, 129 and 643 (1921) ; 28, 225 (1922).

' Core, A. F., Phil. Mag., 46, 256 (1923).

${ }^{5} \mathrm{~A}$ brief treatment is given by Herzfeld and Smallwood, "Physical Chemistry" by H. S. Taylor, p. 226 (1931).

- Van der Waals, Nobelpreisrede (Leiprig, 1911).

; Volmer, Z. Elektrochem., 35, 555 (1929).

\section{A Method of obtaining Polarized Neutron Beams}

Recent progress in cooling methods have made it possible to obtain temperatures where $k T$ becomes comparable with the energy separation, due to space quantization of nuclear spins in high magnetic fields. ${ }^{1}$ The nuclei will, under such conditions, occupy the lower levels of space quantization more abundantly than the higher ones, provided that temperature equilibrium between the lattice of the cooled substance and its nuclei is obtainable. ${ }^{2}$ This effect is equivalent to a polarization of the nuclei, and might be useful for obtaining highly polarized neutron beams without great loss of intensity.

Should the nuclei in question have a resonance level for absorption of slow neutrons (thus absorption by an $S$-level) it would be expected that the capture cross-section would be very dependent on the relative orientation of the neutron spin and the spin of the capturing nucleus. In the special case of an unexcited nucleus having the spin $1 / 2$ and the compound nucleus-formed by slow neutron capture-the spin 0 , the cross-section should be zero for neutrons the spin of which is parallel to the nuclear spin, whilst it should be normal for neutrons of opposite spin orientation. A substance the nuclei of which are polarized will thus polarize a beam of slow neutrons by selective absorption of one component of spin orientation. The degree of polarization will only depend on the ratio of occupation of the two levels of space quantization.

In the rare case of a group of thermal neutrons principally due to one nuclear resonance level, this method will furnish polarized beams of thermal neutrons, as the method proposed by F. Bloch. ${ }^{3}$ In most cases, however, only neutrons belonging to one particular resonance level-thus neutrons having energies of several volts-will be polarized. Precession experiments with such neutrons will permit of measurements of their velocity.

Added in proof: Halpern and Johnson ${ }^{4}$ point out that some paramagnetic salts could give higher magnetic scattering cross-sections for slow neutrons than iron, the atomic factor being bigger. At ordinary temperatures scattering of slow neutrons by paramagnetic salts will not furnish polarized neutron beams. It might, however, be useful to employ for the polarization method proposed by F. Bloch ${ }^{3}$ paramagnetic salts magnetized at very low temperatures instead of magnetized iron.

$$
\text { Hans v. HaLban, JUn. }
$$

Institute of Theoretical Physics, Copenhagen. August 14.

${ }^{1}$ Kürti, N. and Simon, F., Proc. Roy. Soc., A, 149. 152 (1935) de Haas, W. J. and Wiersma, E. C., Rapport 7 me Congr. Int. Froid, 267 (1936)

${ }^{2}$ Heitler, W. and Teller, E., Proc. Roy. Soc., A, 155, 629 (1936); Gorter, C. J., Physica, 3, 995 (1936).

${ }^{3}$ Bloch, F., Phys. Rev., 50, 259 (1936).

' Halpern, O., and Johnson, M. H., jun., Phys. Rev., 52, 52 (1937).

\section{Circular Ultra-sonic Grating in Liquids}

A. Žáček and V. Petržílka have recorded observations of radial and torsional vibrations of annular quartz plates cut perpendicular to the optical axis and have found that, with radial oscillations, standing ultra-sonic waves, which can be rendered visible by means of precipitated lycopodium, are formed in the hole in the middle of the crystal plate.

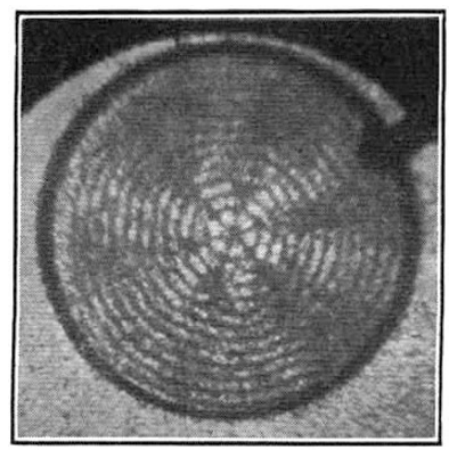

FIG. 1.

A closer investigation of the acoustic field showed that one was dealing with a complicated phenomenon, and that the ultra-sonic waves might be caused not only by radial vibrations but also by other types of vibrations, thus forming interesting figures of lycopodium. This fact was the reason for producing ultra-sonic waves in both gases and liquids, and observing them closely. These experiments have shown that the ultra-sonic waves formed in xylol, or better in a mixture of xylol and carbon tetrachloride, can be observed not only in the hole but also around the crystal plate. In the case of radial vibrations, only concentric circles are produced. If we make use of other types of vibrations, not only concentric circles, but also diametrical lines may appear (Fig. 1). This phenomenon is in all probability connected with the electrical axis of the quartz crystal. The circular grating formed in the liquids by the standing waves will be further utilized in diffraction experiments.

\section{J. ČĚovsKÁ.}

Institute of Physics,

Charles' University, Prague. July 28. 